\title{
Controlled dephasing in single-dot Aharonov-Bohm interferometers
}

\author{
V. Moldoveanu and M. Tolea \\ National Institute of Materials Physics, P.O. Box MG-7, Bucharest-Magurele, Romania \\ B. Tanatar \\ Department of Physics, Bilkent University, Bilkent, 06800 Ankara, Turkey
}

(Received 3 April 2006; revised manuscript received 14 September 2006; published 8 January 2007)

\begin{abstract}
We study the Fano effect and the visibility of the Aharonov-Bohm oscillations for a mesoscopic interferometer with an embedded quantum dot in the presence of a nearby second dot. When the electron-electron interaction between the two dots is considered the nearby dot acts as a charge detector. We compute the currents through the interferometer and detector within the Keldysh formalism and the self-energy of the nonequilibrium Green's functions is found up to the second order in the interaction strength. The current formula contains a correction to the Landauer-Büttiker formula. Its contribution to transport and dephasing is discussed. As the bias applied on the detector is increased, the amplitude of both the Fano resonance and Aharonov-Bohm oscillations are considerably reduced due to controlled dephasing. This result is explained by analyzing the behavior of the imaginary part of the interaction self-energy as a function of energy and bias. We emphasize as well the role of the ring-dot coupling. Our theoretical results are consistent with the experimental observation of Buks et al. [Nature 391, 871 (1998)].
\end{abstract}

DOI: 10.1103/PhysRevB.75.045309

PACS number(s): 73.23.Hk, 85.35.Ds, 85.35.Be, 73.21.La

\section{INTRODUCTION}

The coherent nature of electronic transport through Aharonov-Bohm rings with embedded quantum dots (QD) was clearly established in various experiments. ${ }^{1-4}$ In particular the periodic Aharonov-Bohm oscillations (ABO) of the interferometer conductance and the mesoscopic Fano effect were systematically observed and are nowadays well understood theoretically, at least for noninteracting dots or within mean-field approaches. ${ }^{5-12}$ A more subtle problem in electronic interferometry is related to the decoherence effects caused by inelastic processes like electron-electron interaction, electron-phonon or electron-photon coupling. More generally (see Refs. 13 and 14) the decoherence appears due to the mutual interaction between a coherent system and its environment and leads to a loss of quantum interference between different electronic trajectories.

A particular type of decoherence is the so-called controlled dephasing introduced by Gurvitz ${ }^{15}$ for a double dot system. Using one of the dots as a charge detector Gurvitz proved that the off-diagonal elements of the reduced density matrix of the measured dot are damped. Otherwise stated, the dot coherence is destroyed during the measurement process. Following this idea Buks et al. ${ }^{16}$ have patterned an Aharonov-Bohm interferometer (ABI) with a quantum point contact (QPC) located near the quantum dot. The two subsystems were not coupled directly so their mutual coupling comes only from the Coulomb interaction between electrons in the dot and in QPC. It was found that the transmission through the latter $\mathcal{T}_{\mathrm{QPC}}$ increases smoothly as the plunger gate potential $V_{g}$ applied on the dot increases. Also, whenever a resonant conductance peak of the quantum dot is being scanned the QPC "feels" the passing of a charge carrier through the dot and is therefore called "which path detector" (WPD). Conversely, the current flowing through the WPD induces a reduction of the Aharonov-Bohm oscillations in the ring when the detector is subjected to a rather large bias.
At small bias instead the visibility of the oscillations is not affected by dephasing. A similar experiment with coupled quantum dots was performed by Sprinzak et al. ${ }^{17}$ with a double quantum dot.

The first theoretical consideration of measurement dephasing in quantum dots coupled to nearby detectors was given by Aleiner et al. ${ }^{18}$ The dephasing rate (i.e., the inverse of the time $t_{d}$ required for the detection of addition processes in the QD) was computed for weak mixing between the scattering states describing the WPD. A similar result was obtained by Levinson for a single-level isolated QD coupled to a conducting WPD (Ref. 19) using the influence functional method. ${ }^{20}$ Another treatment proposed by Hackenbroich ${ }^{7}$ is based on the master equation techniques. The reduced density matrix of an isolated quantum dot coupled to a WPD is shown to have modified off-diagonal elements and the dephasing rate was computed within the Markov approximation, taking into account the phase change of the QPC $S$ matrix when one electron enters the QD.

An alternative view on dephasing in Coulomb-coupled mesoscopic conductors was developed by Büttiker et al. ${ }^{21}$ The electron-electron interaction is described by geometrical capacitances and the dephasing rate is given in terms of the voltage fluctuations in QD and WPD.

In a recent work Silva and Levit ${ }^{22}$ presented a detailed analysis of the dephasing rate for a quantum dot perturbed by a WPD by computing the interaction self-energy up to the second order in the Coulomb coupling constant. In the zero temperature limit the dependence of the dephasing rate $\nu$ on the ratio $\mathrm{eV} / \Gamma$ ( $\mathrm{eV}$ being the bias applied to the detector and $\Gamma$ the lead coupling) was discussed. Moreover, in a recent work ${ }^{23}$ it has been found that the dephasing rate of a quantum dot coupled to a detector is maximum at the resonance of the latter.

Although the experiment of Buks et al. has triggered important theoretical developments the main issue of the contributions we just mentioned has been the calculation of the 
dephasing rate for a quantum dot which is isolated or coupled to two leads rather than embedded in a ring. The magnetic field is also absent and, to our best knowledge, no theoretical investigation of dephasing in the specific geometry of Ref. 16 was performed.

Consequently an analysis of the Aharonov-Bohm oscillations depending on the interaction strength or on the WPD bias is not available. Moreover, the recent observation of the mesoscopic Fano effect in single-dot ABI (Ref. 4) poses naturally the problem of possible dephasing effects on the Fano interference.

In this work we consider an Aharonov-Bohm interferometer with an embedded dot which is coupled via a Coulomb term to a second dot playing the role of WPD. We take into account the geometry of the whole system (ring+dot + detector) and compute the currents through the interferometer and WPD within the Keldysh formalism. In particular the interferometer is a many-level system and therefore the current formula is not as simple as for a single-site dot. Following Ref. 22, we compute the first two contributions to the interaction proper self-energies. Our calculations present clear plots showing the effect of dephasing on the mesoscopic Fano line. We capture as well the suppression of the $\mathrm{AB}$ oscillations when a large bias is applied to the detector and discuss the conditions required for the observation of the controlled dephasing. We mention here that in a recent paper ${ }^{24}$ Szafran and Peeters presented an interesting effect in a two dimensional noninteracting ring without any quantum dot. It was shown that due to the Lorentz force the current is asymmetrically injected and that the Aharonov-Bohm oscillations are also affected.

Let us discuss now the approximations we have used in the calculations. A full treatment of the electron-electron interaction would require one to take into account as well the intradot Coulomb interaction and the spin degree of freedom. We argue below that in the absence of the spin-flip effects and in the regime of weak ring-dot the intradot interaction does not bring new effects that have not been addressed before in the existing literature. In a recent paper Jiang et al. ${ }^{25}$ claimed that the intradot interaction does not lead to dephasing while König and Gefen ${ }^{26}$ predicted that the spin-flip processes are responsible for the observed asymmetry of the Aharonov-Bohm oscillations. ${ }^{27}$ The conditions for which a spin pair state is entangled in the dot are rather unlikely to meet in experiments and the data provided by Buks et al. do not reflect such a contribution to dephasing. Another situation in which the intradot interaction and the spin degree of freedom can lead to interesting features is the Kondo regime of the embedded dot (see Ref. 28 for experimental results). This problem was studied by Silva and Gefen, ${ }^{29}$ the main result being that the Kondo correlations reduce the decoherence, as the transport in this case is due to spin fluctuations which are not detected by the charge detector. Further theoretical investigation of the decoherence in the Kondo regime was reported in Ref. 30. However, in the experimental setup of Buks et al. the weak ring-dot coupling prevents the formation of the Kondo correlated state and therefore we believe that neglecting this mechanism is permitted.

The outline of the paper is as follows. Section II settles the notation and gives the relevant formulas for currents and

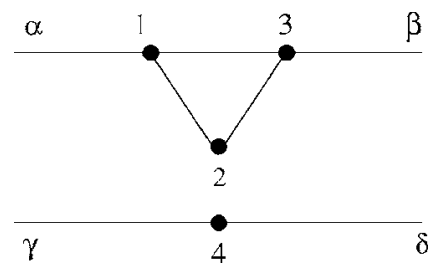

FIG. 1. The interferometer-detector system. The quantum dot is described by site 2 which is Coulomb-coupled to the single-site detector 4 .

self-energies. Section III presents the numerical results and their discussion. We conclude in Sec. IV.

\section{THE MODEL AND THE CURRENT FORMULA}

\section{A. The current formula}

We describe our system by a tight-binding Hamiltonian and consider in a two-lead geometry a simple interferometer composed of three sites, one of them simulating the quantum dot. The "which path detector" is a single site coupled also to two leads (see Fig. 1 for geometry and notation). The full Hamiltonian reads:

$$
\begin{gathered}
H(t)=H_{0}+\chi_{\eta}(t)\left(H_{i}+H_{t}\right), \\
H_{0}=H_{I}+H_{D}+H_{L},
\end{gathered}
$$

where the unperturbed Hamiltonian $H_{0}$ contains the Hamiltonians of the interferometer $I$, detector $D$, and leads $L$. The last two terms in Eq. (1) describe the interferometer-detector interaction and their coupling to the leads. The two perturbations are adiabatically applied, more precisely the switching function $\chi_{\eta}(t)$ is defined such that $\chi_{\eta}(t)=e^{\eta t}$ for $t<0$ and $\chi_{\eta}(t)=1$ for $t>0 . \eta$ is a small positive adiabatic parameter. The adiabatic switching of both the lead-system coupling and interaction eliminates the complications due to the Matsubara complex time contour which would otherwise appear in the perturbation theory for the Keldysh-Green functions. ${ }^{31,32}$ Physically this procedure means that the initial correlations are not taken into account. ${ }^{32}$ The explicit form of the Hamiltonians are as follows:

$$
\begin{gathered}
H_{I}=\sum_{i=1}^{3}\left(\varepsilon_{i}+\delta_{i 2} V_{g}\right) d_{i}^{\dagger} d_{i}+\sum_{i \neq j, i, j=1}^{3} e^{i \varphi_{i j}} t_{i j} d_{i}^{\dagger} d_{j}, \\
H_{D}=\varepsilon_{4} d_{4}^{\dagger} d_{4}, \quad H_{i}=U d_{2}^{\dagger} d_{2} d_{4}^{\dagger} d_{4}, \\
H_{L}=\sum_{\nu} \sum_{n=0}^{\infty}\left[\varepsilon_{n} c_{n \nu}^{\dagger} c_{n \nu}+t_{L}\left(c_{n \nu}^{\dagger} c_{(n+1) \nu}+\text { H.c. }\right)\right], \\
H_{t}=t_{L I}\left(d_{1}^{\dagger} c_{0 \alpha}+d_{3}^{\dagger} c_{0 \beta}\right)+t_{L D}\left(d_{4}^{\dagger} c_{0 \gamma}+d_{4}^{\dagger} c_{0 \delta}\right)+\text { H.c. },
\end{gathered}
$$

$d_{i}, d_{i}^{\dagger}$ are annihilation/creation operators in the interferometer $(i=1,2,3)$ and detector $(i=4)$. Similarly we have on leads the pair $c, c^{\dagger}$. In the leads' Hamiltonian $H_{L}, \nu$ is the lead index (i.e., $\nu=\alpha, \beta, \gamma, \delta$ ) and the parameter $t_{L}$ is the hopping 
constant on leads. In Eq. (3) $V_{g}$ simulates the gate voltage applied on the dot and the magnetic flux $\phi$ piercing the ring is included in the Peierls phases $\phi_{i j}$ attached the hopping constants $t_{i j}$. It is expressed in quantum flux units $\phi_{0}$ and specifically we have $\varphi_{12}=\varphi_{23}=\varphi_{31}=2 \pi \phi / 3 \phi_{0}$ and $\varphi_{j i}=\varphi_{j i}^{*}$. $H_{i}$ describes the Coulomb interaction of strength $U$ between the embedded dot and the detector. $H_{t}$ couples the interferometer and the detector to the sites $0 \nu$ of the lead $\nu$. The corresponding hopping constants are $t_{L I}$ and $t_{L D}$. Assuming that a steady-state regime is already achieved at time $t$, the nonequilibrium Green's function formalism gives the current through the lead $\alpha$ (see for example Ref. 33)

$$
\left\langle J_{\alpha}\right\rangle=\frac{e t_{L I}}{\pi \hbar} \int_{-\infty}^{\infty} d E \operatorname{Re} G_{1 \alpha}^{<}(E),
$$

where $G_{1 \alpha}^{<}(E)$ is the Fourier transform of the real-time lesser Green's function:

$$
G_{1 \alpha}^{<}\left(t, t^{\prime}\right)=i\left\langle c_{0 \alpha, H}^{\dagger}\left(t^{\prime}\right) d_{1, H}(t)\right\rangle .
$$

In the above equation $\langle\cdot\rangle$ denotes the statistical average on the fermionic Fock space with respect to the density matrix operator of the unperturbed time-independent Hamiltonian $H_{0}$. The operators are written in Heisenberg picture with respect to $H(t)$. It is well known that the calculation of $G_{1 \alpha}^{<}$ requires the knowledge of the Green-Keldysh function defined as follows:

$$
G_{m n}\left(\tau, \tau^{\prime}\right):=-i\left\langle T_{C}\left(d_{m, H}(\tau) c_{n, H}^{\dagger}\left(\tau^{\prime}\right)\right)\right\rangle,
$$

where $T_{C}$ is the time-ordering operator along the SchwingerKeldysh "contour" $C=\left(-\infty, \max \left\{\tau, \tau^{\prime}\right\}\right] \cup\left[\max \left\{\tau, \tau^{\prime}\right\},-\infty\right)$. The first step of the calculation is to express the mixedindices Green function $G_{1,0 \alpha}$ using its Dyson equation (see Ref. 35). Since the lead $\alpha$ is coupled to the interferometer by a single site we have:

$$
G_{1 \alpha}\left(\tau, \tau^{\prime}\right)=t_{L I} \int_{C} d \tau_{1} G_{11}\left(\tau, \tau_{1}\right) g_{0 \alpha, 0 \alpha}\left(\tau_{1}, \tau^{\prime}\right),
$$

where $g_{0 \nu, 0 \nu}$ is the Green's function of the semi-infinite lead $\nu=\alpha, \beta, \gamma, \delta$ at site 0 . This quantity is known (see Ref. 33):

$$
g_{0 \nu, 0 \nu}^{R}(E)= \begin{cases}\frac{1}{2 t_{L}^{2}}\left(E-i \sqrt{4 t_{L}^{2}-E^{2}}\right) & \text { if } E<\left|2 t_{L}\right|, \\ \frac{1}{2 t_{L}^{2}}\left(E-\sqrt{E^{2}-4 t_{L}^{2}}\right) & \text { if } E>\left|2 t_{L}\right|\end{cases}
$$

in which $t_{L}$ is the hopping constant on leads. Also one has:

$$
g_{0 \nu, 0 \nu}^{<}(E)=2 \pi i f^{\nu}(E) \rho(E)=-2 i f^{\nu}(E) \operatorname{Im} g_{0 \nu, 0 \nu}^{R}(E) .
$$

Here $f^{\nu}(E)$ is the Fermi function for the lead $\nu$ and $\rho$ is the electronic density at the site $0 \nu$ of the lead. Note that since we take the same hopping constant on leads $\rho$ does not depend on $\nu$. Expressing the contour integral in Eq. (10) via the Langreth rules ${ }^{34}$ and plugging its Fourier transform into Eq. (7) one gets after simple calculations the following current formula (we shall omit the energy dependence when this causes no confusion):

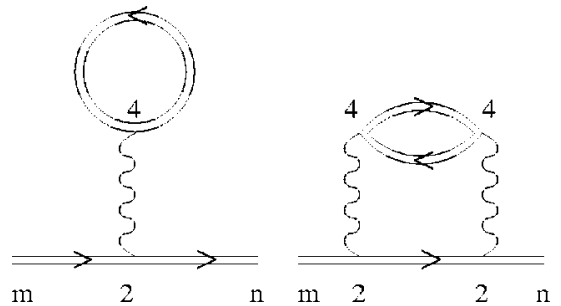

FIG. 2. The first two contributions to self-energy $\Sigma_{i}^{1}$ (left) and $\Sigma_{i}^{2}$ (right).

$$
\left\langle J_{\alpha}\right\rangle=-\frac{e t_{L I}^{2}}{\hbar} \int d E \rho \operatorname{Im}\left(2 G_{11}^{R} f^{\alpha}+G_{11}^{<}\right) .
$$

Note that due to the leads' density of states $\rho$ [see Eq. (12)] the integral runs only over the continuous spectrum of the leads $\left[-2 t_{l}, 2 t_{L}\right]$. The current formula Eq. (13) reduces the problem to the calculation of a matrix element of the interferometer Green's function. This can be done using the Feynman-Dyson expansion along the contour $C$. Without giving the straightforward details we state that the contour Green's function which is a $4 \times 4$ matrix satisfies the equation:

$$
G=G_{\text {eff }}+G_{\text {eff }} \Sigma_{i} G,
$$

where $\Sigma_{i}$ is the self-energy due to interaction and the effective Green function describes the noninteracting system in the presence of the leads, i.e.,

$$
G_{\text {eff }}=G_{0}+G_{0} \Sigma_{l} G_{\text {eff }} .
$$

Here $G_{0}$ describes the noninteracting decoupled system $(\mathrm{QD}+\mathrm{QPC})$ and $\Sigma_{l}$ is the usual self-energy of the leads:

$$
\Sigma_{l, m n}(z)=\left\{\begin{array}{cc}
t_{L}^{2} g_{0 \nu, 0 \nu}(z) & \text { if } m=n=1,3, \\
t_{L}^{2}\left(g_{0 \gamma, 0 \gamma}(z)+g_{0 \delta, 0 \delta}(z)\right) & m=n=4, \\
0 \text { if } m \neq n & \text { or } m=n=2 .
\end{array}\right.
$$

\section{B. The self-energies}

In what concerns the interaction self-energy $\Sigma_{i}$ we compute only the first two contributions $\Sigma_{i}^{1}$ and $\Sigma_{i}^{2}$ which can be identified from the diagrams in Fig. 2. We used double lines for electronic propagators since they are "dressed" by the leads' self energy. Using the Langreth rules for dealing with time-integrals and performing the Fourier transform one obtains for $m, n=1,2,3$,

$$
\begin{gathered}
\sum_{i, m n}^{R, 1}(E)=-\delta_{m 2} \delta_{n 2} i \frac{U}{2 \pi} \int d E^{\prime} G_{\mathrm{eff}, 44}^{<}\left(E^{\prime}\right), \quad \Sigma_{i, m n}^{<, 1}(E)=0, \\
\Sigma_{i, m n}^{<, 2}(E)= \\
\delta_{m 2} \delta_{n 2} \frac{U^{2}}{2 \pi^{2}} \int d E_{1} d E_{2} G_{\mathrm{eff}, 22}^{<}\left(E-E_{1}\right) \\
\times G_{\mathrm{eff}, 44}^{<}\left(E_{2}\right) G_{\mathrm{eff}, 44}^{>}\left(E_{2}-E_{1}\right) .
\end{gathered}
$$

The explicit forms for $\Sigma_{i, 44}^{R, 1}, \Sigma_{i, 44}^{R, 2}$, and $\Sigma_{i, 44}^{R,<}$ are similar, the 
only difference being that $G_{\text {eff, } 22}$ and $G_{\text {eff, } 44}$ have to be interchanged. The retarded self-energy is related to the lesser and greater self-energies by the identity (see Ref. 22):

$$
\Sigma_{i}^{R, 2}(E)=\lim _{\epsilon \rightarrow 0+} \frac{i}{2 \pi} \int d E^{\prime} \frac{\Sigma_{i}^{>, 2}\left(E^{\prime}\right)-\Sigma_{i}^{<, 2}(E)}{E-E^{\prime}+i \epsilon} .
$$

To obtain the greater self-energy $\Sigma_{i, m n}^{>, 2}$ one has just to interchange $>$ and $<$. The Dyson and Keldysh equations for the retarded and lesser Green's functions read:

$$
\begin{gathered}
G^{R}=G_{\text {eff }}^{R}+G_{\text {eff }}^{R} \Sigma_{i}^{R} G^{R}, \\
G^{<}=\left(1+G^{R} \Sigma_{i}^{R}\right) G_{\text {eff }}^{<}\left(1+\Sigma_{i}^{A} G^{A}\right)+G^{R} \Sigma_{i}^{<} G^{A}, \\
G_{\text {eff }}^{<}=G_{\mathrm{eff}}^{R} \Sigma_{l}^{<} G_{\mathrm{eff}}^{A} .
\end{gathered}
$$

The last identity uses that $\left(G_{0}^{R}\right)^{-1} G_{0}^{<}=0$. A simple calculation gives alternative forms of (14) and (21):

$$
\begin{aligned}
& G=G_{0}+G_{0}\left(\Sigma_{l}+\Sigma_{i}\right) G, \\
& G^{<}=G^{R}\left(\Sigma_{l}^{<}+\Sigma_{i}^{<}\right) G^{A} .
\end{aligned}
$$

In the following we shall use the perturbative results from above to compute the current $\left\langle J_{\alpha}\right\rangle$ via Eq. (13). Using the identity $G^{R}-G^{A}=2 i G^{R} \operatorname{Im} \Sigma^{R} G^{A}$, Eq. (24) and the explicit expression for $\Sigma_{l, 11}$ and $\Sigma_{l, 33}$, a straightforward algebra gives:

$$
\begin{aligned}
\left\langle J_{\alpha}\right\rangle= & \frac{e t_{L I}^{2}}{\hbar} \int_{-2 t_{L}}^{2 t_{L}} d E\left(2 \pi \rho^{2} G_{13}^{R} G_{31}^{A}\left(f^{\alpha}-f^{\beta}\right)\right. \\
& \left.-\rho G_{12}^{R} \operatorname{Im}\left(2 \Sigma_{i, 22}^{R} f^{\alpha}+\Sigma_{i, 22}^{<}\right) G_{21}^{A}\right):=J_{1}+J_{2} .
\end{aligned}
$$

The first term $J_{1}$ from Eq. (25) is clearly a Landauer-type current. The second term in the current formula is a correction to the Landauer formula due to interaction and was not considered in previous papers. ${ }^{18,22}$ Equation (25) is a central result of the present work. In the following we provide further supporting arguments for its validity.

As emphasized in the seminal paper of Meir and Wingreen $^{36}$ the Landauer formula holds also for interacting systems in linear regime at zero temperature. The argument is based on the general result of Luttinger ${ }^{37}$ according to which the imaginary part of the self energy vanishes at the Fermi level, in all orders of the perturbation theory. The subtle point here is that if one of the two interacting subsystems (i.e., the detector) is submitted to a finite bias one gets a nonvanishing imaginary part of the self energy even in the limit $T \rightarrow 0$. The explicit calculation in Ref. 22 for the case of a single-site dot coupled to a quantum point contact clearly shows this fact. As we shall see in Sec. III the correction to the Landauer formula contributes nontrivially to transport and therefore should be kept in the current formula. As a consequence, the total current cannot be expressed in terms of the QD transmittance. We point out that in Ref. 22 the starting point is a non-interacting formula for the QD transmittance $t_{\mathrm{QD}}$ which involves only the retarded Green's function. The latter in turn contains the self-energies due to both e-e interactions and lead-dot coupling. However, König and Gefen ${ }^{26}$ argued recently that such a formula may not properly define the transmission amplitude of interacting quantum dots. In spite of the fact that even in the interacting case the integrand of $J_{1}$ resembles a transmission amplitude the presence of the correction due to the $\operatorname{Im} \Sigma^{R}$ and $\operatorname{Im} \Sigma^{<}$ prevents one to define safely $t_{\mathrm{QD}}$. The rest of the paper will be mainly devoted to the analysis of $J_{1}$ and $J_{2}$.

The current through the lead $\gamma$ is obtained in a similar way. We do not give its expression here but we note that the first order self-energy felt by the detector $\sum_{i, 44}^{R, 1}$ $=-\frac{i}{2 \pi} U \int d E G_{\mathrm{eff}, 22}^{<}(E)=U\left\langle n_{22}\right\rangle$ where $\left\langle n_{22}\right\rangle$ is the electronic occupation number of the noninteracting dot. Then clearly the WPD Green functions record the sudden change of $\left\langle n_{22}\right\rangle$ by one, corresponding to addition of one electron to + the QD.

Although the self-energy expressions are not simple the calculations can be eventually performed since the effective lesser Green functions are given in Eq. (22) and the retarded functions can be computed since they describe noninteracting systems $(m, n=1,2,3)$. Then clearly, one has to invert finite rank non-hermitian matrices and perform the energy integrals from Eqs. (17)-(19) to get the self-energies expression. After some straightforward calculations the retarded self-energy of the dot reads:

$$
\begin{aligned}
\Sigma_{i, 22}^{R, 2}(E)= & \frac{i}{2 \pi} t_{L D}^{4} U^{2} \int d E^{\prime} d E^{\prime \prime} \tilde{\rho}_{44}\left(E^{\prime \prime}\right)\left(\frac{G_{\mathrm{eff}, 22}^{>}\left(-E^{\prime}\right)}{E+E^{\prime}-E^{\prime \prime}+i \delta}\right. \\
& \left.-\frac{G_{\mathrm{eff}, 22}^{<}\left(E^{\prime}\right)}{E-E^{\prime}+E^{\prime \prime}+i \delta}\right),
\end{aligned}
$$

where the generalized spectral density:

$$
\begin{aligned}
\tilde{\rho}_{44}(E):= & \sum_{\nu, \nu^{\prime}=\gamma, \delta} \int d E_{1} d E_{2} \delta\left(E_{1}-E_{2}-E\right) \rho\left(E_{1}\right) \rho\left(E_{2}\right) f^{\nu^{\prime}}\left(E_{2}\right) \\
& \times\left(1-f^{\nu}\left(E_{1}\right)\right)\left|G_{\mathrm{eff}, 44}^{R}\left(E_{1}\right)\right|^{2}\left|G_{\mathrm{eff}, 44}^{R}\left(E_{2}\right)\right|^{2} .
\end{aligned}
$$

This result is similar to Eq. 15 from Ref. 22 We have however extra terms coming from the more complex geometry and the spectral density $\widetilde{\rho}_{44}$ is far more complicated than the one given in Eq.16 from Ref. 22. Now one should use Eq. (22) to express the effective Green's functions. Explicit expressions for the imaginary and real parts of $\Sigma_{i, 22}^{R, 2}$ are found by virtue of the principal-value formula. One can show without much effort that in the zero temperature limit $\operatorname{Im} \sum_{i, 22}^{R, 2}(E)=0$ if there is no bias applied on the WPD (i.e., $\mu_{\delta}=\mu_{\gamma}$ ). Thus, there will be no dephasing as long as the detector is in equilibrium.

At this level of approximation the retarded Green's function of the interferometer is known from the Dyson equation once we have computed all the effective Green's functions and the first two contributions to interaction self-energy. It is now a usual Green's function associated with a single particle operator

$$
\begin{gathered}
G_{44}^{R}(E)=\left\langle 4\left|\left(H_{D}-\Pi_{D}\left(\Sigma_{l}^{R}+\Sigma_{i}^{R}\right) \Pi_{D}-E\right)^{-1}\right| 4\right\rangle, \\
G_{m n}^{R}(E)=\left\langle m\left|\left(H_{I}-\Pi_{I}\left(\Sigma_{l}^{R}+\Sigma_{i}^{R}\right) \Pi_{I}-E\right)^{-1}\right| n\right\rangle,
\end{gathered}
$$

where $\Pi_{D}, \Pi_{I}$ are projection operators onto their associated single particle Hilbert spaces. More explicitly, $\Pi_{D}=|4\rangle\langle 4|$ 
and $\Pi_{I}=\sum_{i=1}^{3}|i\rangle\langle i|$. Also, the self-energies are now associated with the operators $\Sigma(E):=\Sigma_{i, j=1}^{4} \Sigma_{i j}(E)|i\rangle\langle j|$. Then following the same steps as in Refs. 11 and 12 we use the Feschbach formula to rewrite $G_{m n}^{R}$ in a more useful form:

$$
G_{m n}^{R}(z)=\left\langle m\left|G_{R}^{R}(z)+H_{R D} G_{D}^{R}(z) H_{D R}\right| n\right\rangle,
$$

where $H_{R D}$ and $H_{D R}$ are transfer terms between the dot and the ring (i.e., $\left.H_{R D}=t_{12} e^{i \varphi_{12}}|1\rangle\left\langle 2\left|+t_{32} e^{i \varphi_{32}}\right| 3\right\rangle\langle 2|\right)$ and $G_{R}^{R}$ $=\left(H_{R}-\Pi_{I} \Sigma_{l}^{R}(z) \Pi_{I}-z\right)^{-1}$ describes the ring without the dot in the presence of the two leads $\alpha, \beta$. $G_{D}$ is the effective Green function of the dot $\left(H_{d}\right.$ is the single-particle Hamiltonian of the noninteracting uncoupled dot):

$$
G_{D}^{R}(z)=\left(H_{d}-\Pi_{I} \Sigma_{i}^{R}(z) \Pi_{I}-\Sigma_{d}(z)-z\right)^{-1},
$$

where $\Sigma_{d}:=H_{D R} G_{R}^{R}(z) H_{R D}$ is a noninteracting effective selfenergy due to the ring-dot coupling. Obviously, this quantity does not depend on bias and can be computed explicitly by writing the $2 \times 2$ matrix $G_{R}^{R}$. We shall need $\Sigma_{d}$ in our calculations presented in Sec. III. The advantage of having $G^{R}$ in this form is twofold. First, the interaction self-energy appears naturally only in the dot's Green's function. Second, the resonant processes involving the dot are collected in the second term and clearly separated from the noninteracting coherent background contribution of the reference arm of the interferometer.

\section{RESULTS AND DISCUSSION}

Let us first set some notation for the parameters that will be used throughout this section. We work always with a weak ring-dot coupling and put $t_{12}=t_{23}:=t_{R D}$. The leads are instead strongly coupled to the detector and interferometer. As the energy unit we choose the hopping constant on leads $t_{L}$. Consequently the gate potential, the energy, self-energies, and the bias are to be understood in $t_{L}$ units while the current will be given in $e t_{L} / h$ units. The results do not depend on the value of $t_{L}$ so we choose for simplicity $t_{L}=1$. The on-site energy of the leads is chosen as the energy reference. The bias on the interferometer is denoted by $V_{0}$ and the bias on the detector by $V$. We apply both biases in a symmetric way. More precisely, the chemical potentials $\mu$ of the unbiased leads attached to the interferometer and detector are shifted by $\pm V / 2$ and $\pm V_{0} / 2$, respectively.

Before investigating the effect of the electron-electron interaction on the $\mathrm{AB}$ oscillations let us look at the Fano line shapes of the current $J_{\alpha}$. Figure 3 shows the behavior of the Fano resonance when the bias $V$ on the detector increases, for two values of the interaction strength $U=0.3$ and $U$ $=0.5$. The magnetic flux is fixed at $\phi=0.0$ and the bias $V_{0}$ $=0.1$. Other parameters are given in the figure caption. There are two features to observe: (i) the amplitude of the Fano line decreases for larger bias and (ii) when $U=0.5$ the Fano line is shifted with respect to the resonance at $U=0.3$. The reduction of the Fano line is more pronounced for $U=0.5$. The inset in Fig. 3 gives the current $J_{\gamma}$ through the detector as a function of the gate potential $V_{g}$ applied on the dot at interaction $U=0.5$. We take the on-site energies $\varepsilon_{i}=0$ for $i$ $=1,2,3$ and $\varepsilon_{4}=0.1$. Clearly the detector feels the electrons

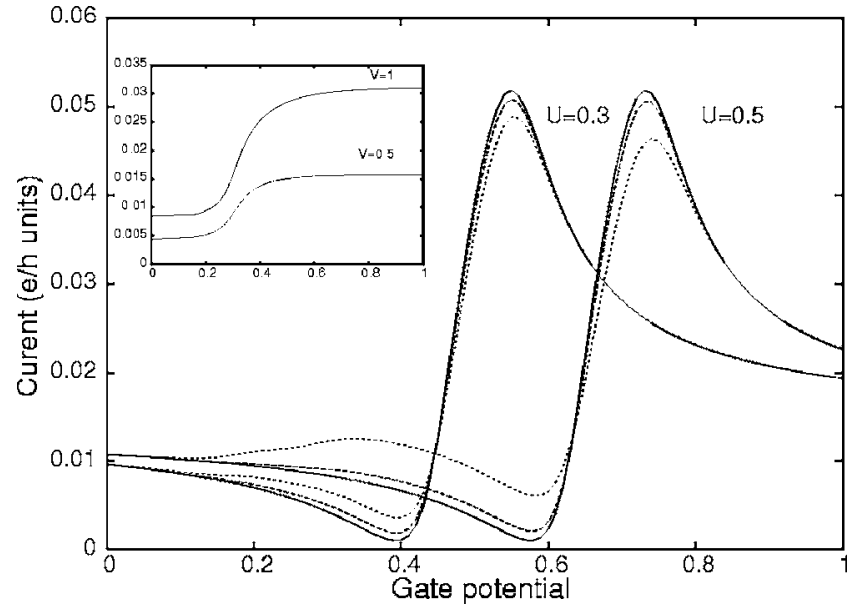

FIG. 3. Coulomb-modified Fano line shapes as a function of the gate potential $V_{g}$ (in units of $t_{L}$ ) at different bias on the detector; solid line $V=0.0$, dashed line $V=0.5$, dotted line $V=1.0$. Inset: the detector response at $U=0.5$. Other parameters: $t_{R D}=0.3, t_{L D}=1$, $t_{L I}=0.8, t_{L}=1, \mu=0$.

passing through the embedded dot. The height of the current step is a measure of the detector sensitivity to the electrons crossing the embedded dot. The inset shows that as the bias increases the response of the detector is enhanced. As we shall show below this suggests that the quantum interference within the interferometer is more affected by the electronelectron interaction at large bias. The current step does not follow precisely the Fano resonance for $U=0.5$ because in our approximation the first order self-energy of the detector $\sum_{i, 44}^{R, 1}$ is computed in terms of the occupation number of the noninteracting dot $\left\langle n_{22}\right\rangle$ (this quantity increases by one as the embedded dot accumulates one electron). For this reason the steplike behavior of $J_{\gamma}$ is correlated with the noninteracting Fano line which is located to the left of the interacting one. A better correspondence between the detector characteristics and the Fano line can be achieved within a self-consistent calculation. Here we prefer to keep the lowest approximation and look for the role of the second order self-energy in transport. We note also that the positions of the Fano line and of the jump in the detector signal are not strictly around the eigenvalue $\varepsilon_{2}$ of the isolated dot. As shown in Eq. (30) even in the noninteracting case the effective Green's function of the embedded dot has a pole whose real part does not have to coincide with $\varepsilon_{2}$. This is due to the ring-dot coupling selfenergy $\Sigma_{d}$ which brings a shift in the real part of the resonance (for more discussion of this point we refer to Ref. 11).

The two currents from Eq. (25) are compared in Fig. 4 which is a zoom from the Fano dip shown in Fig. 3 at $U$ $=0.3$. The correction $J_{2}$ to the Landauer current $J_{1}$ takes significant values only around the Fano resonance. $J_{1}$ shows a dip structure depending very weakly on bias. In contrast, $J_{2}$ increases with $V$ and there is a critical bias $V_{c}$ such that if $V>V_{c}$ then $J_{2}>J_{1}$. The numerical calculations show therefore that in the neighborhood of the Fano resonance $J_{2}$ cannot be neglected and also that this correction is responsible for the enhanced Fano dip seen in Fig. 3. This means that $J_{2}$ diminishes the whole Fano line, hence it affects its visibility.

The $\mathrm{AB}$ oscillations appear when the magnetic field varies and the gate potential is fixed to a value from the range 


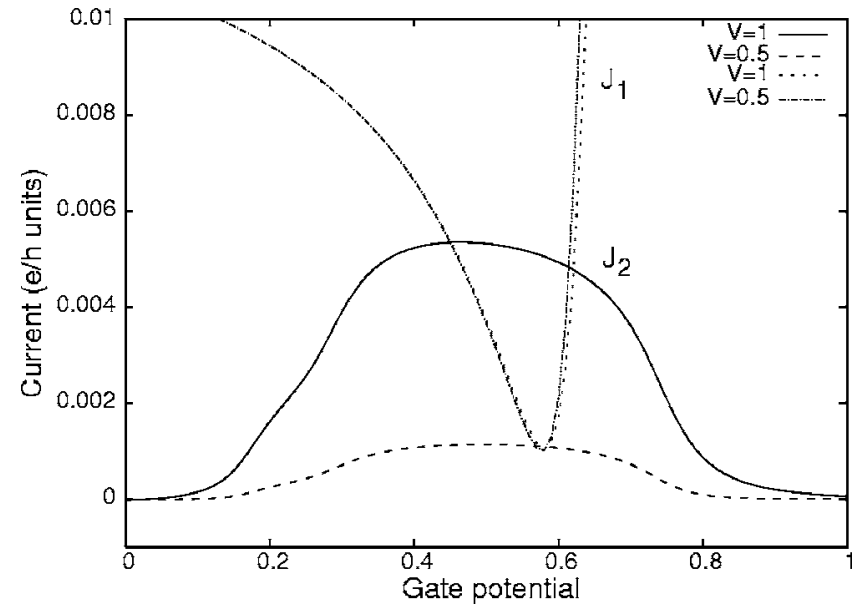

FIG. 4. The Landauer-like current $J_{1}$ (dotted and dash-dotted lines) and the correction $J_{2}$ (solid and long-dashed line) around the Fano dip. $J_{1}$ changes negligibly with increasing the bias $V$ while $J_{2}$ increases strongly and even exceeds $J_{1}$ at $V=1.0$. The gate potential is given in $t_{L}$ units. Other parameters: $t_{L}=1, \mu=0$.

where the Fano line develops. Figure 5 shows the reduction of the $\mathrm{AB}$ oscillation amplitude as the bias on the detector $V$ increases. In Fig. 5(a) we plot oscillations around the Fano peak $\left(V_{g}=0.55, U=0.3\right)$ from Fig. 3. The dephasing appears already at small bias $V=0.25$ and is considerably enhanced at large bias. At $V=1$ the $\mathrm{AB}$ oscillation amplitude is reduced roughly by $50 \%$. A slight asymmetry with respect to $\phi=0$ is noticed. Around the Fano peak the dephasing mainly affects the $\mathrm{ABO}$ maxima and is due to the reduction in the Landauer like current $J_{1}$. Interestingly, the oscillations taken near the Fano dip $V_{g}=0.4$ [see Fig. 5(b)] are more damaged because their minima are pushed upward. This is due to the increasing contribution of $J_{2}$. In particular, around integer values of the magnetic flux the reduction of the maxima and minima are comparable. A similar dephasing effect is obtained for a gate potential associated with the middle of the Fano line

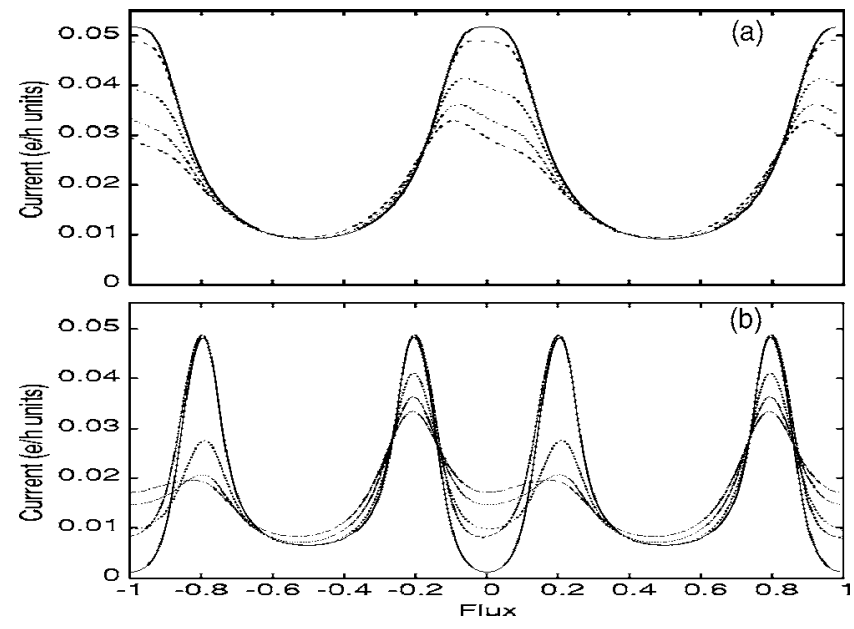

FIG. 5. The reduction of the $\mathrm{AB}$ oscillations with increasing bias on the detector for two values of the gate potential (a) $V_{g}=0.55$ and (b) $V_{g}=0.40$ (in $t_{L}$ units). From top to bottom the biases are $V$ $=0.00, V=0.25, V=0.50, V=0.75, V=1.00$ (in $t_{L}$ units). Other parameters: $t_{L}=1, \mu=0$.

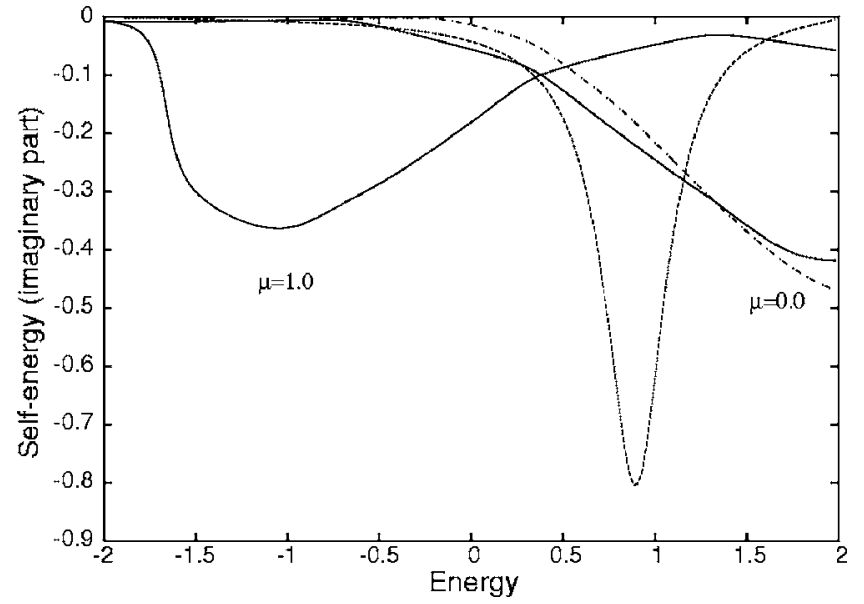

FIG. 6. The imaginary part of the self-energies $\boldsymbol{\Sigma}_{i, 22}^{R, 2}$ and $\Sigma_{d}$ as a function of energy $E$ for fixed bias $V_{0}=0.1$ and chemical potential $\mu . \operatorname{Im} \Sigma_{d}$ is plotted with dashed line and depends only on energy. At $\mu=0.0$ two plots are shown for $\operatorname{Im} \Sigma_{i, 22}^{R, 2}: V=1.0$ - full line and $V$ $=0.5$ dashed-dotted line. For $\mu=1.0$ the full line corresponds to $V$ $=1.0$. All quantities are given in $t_{L}$ units and $t_{L}=1$.

(not shown). The above observations show that both currents contain the dephasing effect due to electron-electron interaction and suggest that in order to detect experimentally the dephasing in such systems one should look around the Fano dip because here the effect is stronger. The double-maxima aspect of the $\mathrm{AB}$ oscillations in Fig. 5(b) is not an interaction effect and it is easy to explain. As known, the energy levels of the ring behave like $\sin \phi$. Single-maxima AB oscillations are obtained when the energy of the incident electron $E$ is close to a ring level while the double oscillations appear when $E$ crosses it twice in flux period (see examples in Ref. 38).

In the following we investigate the conditions under which the suppression of the $\mathrm{AB}$ oscillations due to the dotdetector interaction is discerned. To this end we compare the imaginary parts of the two self-energies appearing in the effective Green's function of the embedded dot [see Eq. (30)]. Note that $\Sigma_{d}$ appears only in the Landauer-like current and that $\sum_{i, 22}^{R, 1}$ is real. At low temperature and small bias applied on the interferometer the relevant range for this comparison is (see the integration limits in the current formula) $[\mu$ $\left.-e V_{0} / 2, \mu+e V_{0} / 2\right]$.

In Fig. 6 we give the imaginary parts of the two selfenergies as a function of energy for different values of bias on the detector. The bias applied on the interferometer is $V_{0}=0.1$ and the magnetic field is fixed at $\phi=0 . \operatorname{Im} \Sigma_{d}$ depends only on the energy of the electrons entering the interferometer while $\operatorname{Im} \Sigma_{i, 22}^{R, 2}$ is very sensitive to both bias $V$, and the chemical potential on leads $\mu$. For $\mu=0$ it nearly vanishes at energies smaller than -0.5 but increases as $E$ approaches the upper bound of the lead's spectrum $2 t_{L}$. A first important observation is that in the integration domain $[-0.05,0.05]$ the imaginary parts of $\Sigma_{d}$ and $\Sigma_{i, 22}^{R, 2}$ are comparable. Secondly, we remark that as the bias $V$ increases $\operatorname{Im} \Sigma_{i, 22}^{R, 2}$ exceeds (in absolute value) $\operatorname{Im} \Sigma_{d}$. Then looking at the $\mathrm{AB}$ oscillations from Fig. 5 one infers that the dephasing effect appears as the interaction self-energy is of the same 



FIG. 7. (a) Fano lines at different values of the bias applied on the interferometer; full line $-V_{0}=0.15$, dashed line $V_{0}=0.2$, dotted line $V_{0}=0.25$. (b) The reduction of the Aharonov-Bohm oscillations at $V_{0}=0.25$. $V_{g}=0.52$ corresponds to the Fano dip. Other parameters $U$ $=0.5, t_{R D}=0.3, t_{L D}=1, t_{L I}=0.8, \mu=0$. All quantities are given in $t_{L}$ units and $t_{L}=1$.

order as the effective self-energy due to the ring-dot coupling. The stronger suppression of the $\mathrm{AB}$ oscillation at bias $V=1$ is also understood. We have checked that by varying the magnetic flux the self-energies do not vary drastically and the above discussion still holds.

Another interesting aspect showing the controlled feature of dephasing is revealed when the bias window of the interferometer is shifted by changing the equilibrium chemical potential $\mu$ of the decoupled leads. The left curve in Fig. 6 gives again the function $\operatorname{Im} \sum_{i, 22}^{R, 2}(E)$ at large bias $V=1$ but now for $\mu=1$. Thus, the integration domain is now [0.95, 1.05] and in this region $\operatorname{Im} \Sigma_{i, 22}^{R, 2} \ll \operatorname{Im} \Sigma_{d}$. We found in this case that the dephasing is more difficult to notice. Actually the oscillations of the Fano peak for zero bias and $V=1$ do not differ significantly and a very small dephasing appears when a gate potential around the Fano dip is chosen. Therefore, the dephasing is controlled not only by the bias applied on the environment but also by the properties of the dephased system, i.e., by the behavior of the self-energy $\Sigma_{d}$ which depends in turn on the ring-dot coupling and on energy. We stress that in Ref. 22 the leads' self-energy does not depend on $E$ and the above discussion cannot be made.

Evidently, one has to check whether the above results are still valid at other values for the bias across the interferometer. In Fig. 7(a) we show the Fano line shapes for three values of $V_{0}$. The Coulomb interaction strength is chosen here as $U=0.5$. As $V_{0}$ increases the Fano lines move globally to higher values of the current and are broader. This feature is easy to understand. The main point is that the width of the bias window sets the range of the gate potential for which one can observe the Fano effect. This is because the quantum interference is possible only as long as the discrete level of the embedded dot lies within the bias window. Therefore, at large bias the Fano line has a larger width due to a larger window. In Fig. 7(b) we plot the AB oscillations taken from the Fano dip of the dotted line in Fig. 7(a). As the bias on the detector increases from $V=0.25$ (full line) to $V=0.50$ (dashed line) the dephasing effect develops and is similar to previous plots.

In what concerns the role of the interaction strength it is clear that the dephasing is enhanced as $U$ increases, since the interaction self-energy is directly proportional to $U^{2}$. On the other hand our perturbative approach does not allow large values for $U$. Since the embedded dot and the detector are spatially separated by approximately $1 \mu \mathrm{m}$ we believe that this parameter has also small values in the concrete experimental realization. We also point that in the case where the dephasing is clearly observed (i.e., when $\operatorname{Im} \Sigma_{i, 22}^{R, 2} \sim \operatorname{Im} \Sigma_{d}$ ) the interaction strength $U=0.3$ is much bigger than $\operatorname{Im} \Sigma_{d}$.

Now, let us see what happens when the ring-dot coupling is varied. The analytical expression for $\Sigma_{d}$ shows that it behaves like $t_{R D}^{2}$. Thus, by keeping the bias fixed and decreasing $t_{R D}$ the imaginary part of $\Sigma_{d}$ diminishes. Consequently the ratio $\operatorname{Im} \Sigma_{i, 22}^{R} / \operatorname{Im} \Sigma_{d}$ increases and from the above analysis one expects further reduction of the $\mathrm{AB}$ oscillation. This is precisely demonstrated in Fig. 8 where $\mathrm{AB}$ oscillations at bias $V=0.5$ are given for different couplings. Each oscillation corresponds to the peak of the Fano line at the given $t_{R D}$. Physically the enhanced dephasing noticed at smaller ringdot couplings is understandable because by reducing $t_{R D}$ the dwell time of electrons inside the dot increases and therefore they can be easily detected. We stress that in experiments the ring-dot coupling can be adjusted freely and therefore the

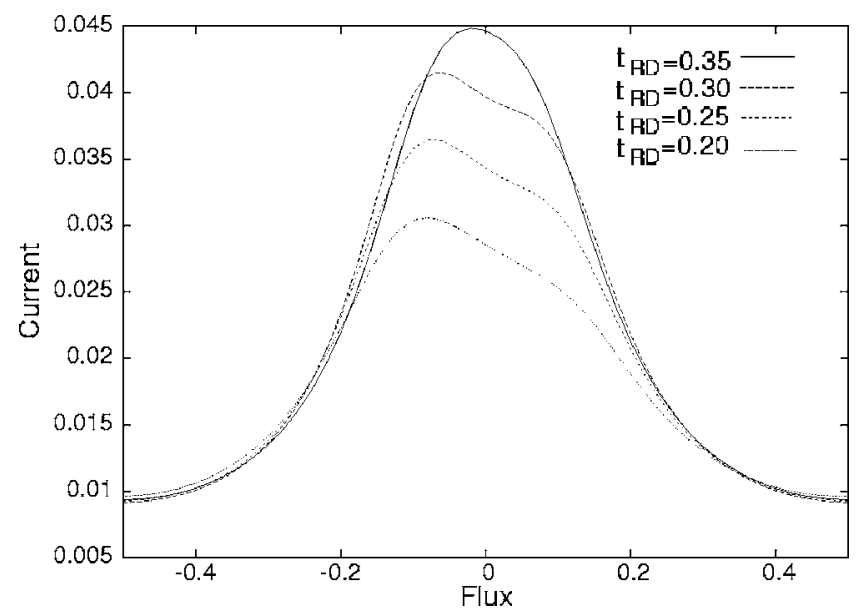

FIG. 8. The effect of the ring-dot coupling on the dephasing. As $t_{R D}$ decreases the $\mathrm{AB}$ oscillations are more damaged. Other parameters: $U=0.3, V_{0}=0.1, t_{L D}=1, t_{L I}=0.8$. 


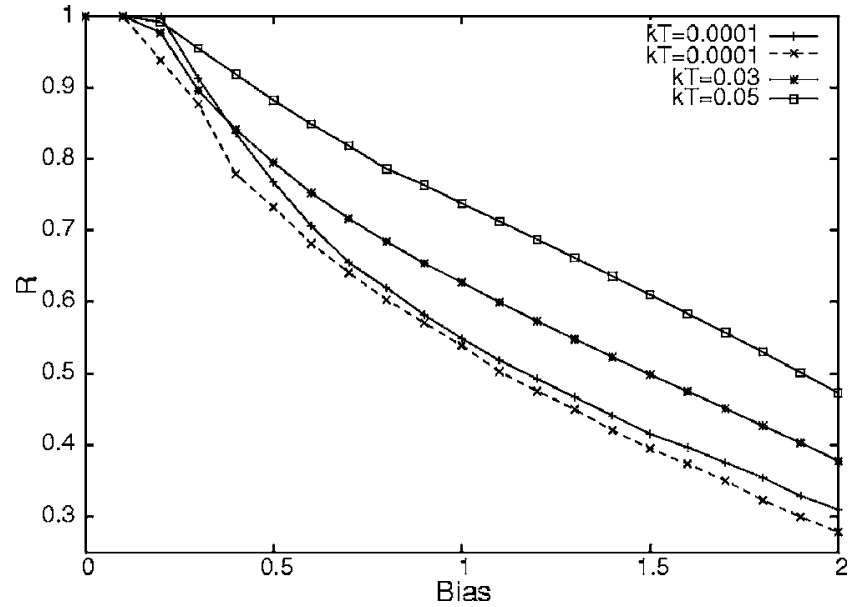

FIG. 9. The ratio $R$ defined in the text as a function of bias for different temperatures. At $k T=0.0001$ the full lines correspond to $\mathrm{AB}$ oscillations of the Fano peak while the dashed line is assigned to the Fano dip. The dephasing reduces as the temperature increases.

dephasing in the system we consider should be easily seen for weak ring-dot couplings.

Further analysis of the controlled dephasing is contained in Fig. 9. It gives the ratio $R=A\left(V_{i}\right) / A_{0}$ between the amplitude of the $\mathrm{AB}$ oscillations at different biases $V_{i}$ and the zero-bias amplitude $A_{0}$. This quantity is somehow similar to the visibility of the oscillations measured in Ref. 16. At small temperatures we give the visibility for two gate potentials corresponding to the Fano peak (full line) and dip (dashed line). The behavior of $R$ confirms that in the presence of mutual Coulomb interaction between the detector and dot the coherence of the interferometer is reduced as the bias increases. This was the main experimentally observed feature in the work of Buks et al. They have found that at small bias the visibility is almost constant while it decreases at higher bias and when the detector response is accurate. We note from Fig. 9 that the visibility of the oscillations taken around the Fano dip decrease faster than the one from the Fano peak. As we explained, this is due to the nonvanishing contribution of the second term in the current formula around the Fano dip.

In order to look for temperature effects on dephasing we have also considered higher temperatures $k T=0.03$ and $k T$ $=0.05$. We have checked that the interaction self-energies do not depend strongly on temperature (not shown). On the other hand, the effective self-energy $\Sigma_{d}$ does not depend on $T$. When the temperature increases the integral in the current formula runs over the whole range $[-2,2]$ and therefore it scans energies where $\operatorname{Im} \Sigma_{i, 22}^{R} \ll \operatorname{Im} \Sigma_{d}$ as well. This could explain the slower decrease of the $\mathrm{AB}$ oscillation visibility noticed for $k T=0.03$ and $k T=0.06$.

Before concluding let us comment on the relation and relevance of our calculations to the experiment of Buks et al. or to future experiments. We have used a detector which has a simple structure (a single level) and differs from the quantum point contact used in experiments. As a consequence we cannot compare directly our results to the experimental plots of Ref. 16. We believe, however, that our simple model captures the main features of the experiment and can stimulate further measurements in order to check the controlled dephasing of the Fano effect. This topic was never considered.

\section{CONCLUSIONS}

Motivated by the experimental paper of Buks et al. ${ }^{16}$ we have looked at the coherent transport properties of an $A B$ interferometer with an embedded quantum dot which interacts with a nearby second dot. The Keldysh formalism gives the current through the ring-dot system and the detector. The interaction self-energy is computed perturbatively up to the second order in the interaction strength. Though the interaction effects on the quantum coherence were discussed in several papers, ${ }^{10,18,21,22,25}$ to our best knowledge this system was not considered theoretically before.

The results we have obtained and discussed within this paper underline that: (i) even in the low-order perturbative approach taken here the electron-electron interaction causes controlled dephasing which manifests in the reduction of Fano line amplitude and the suppression of $\mathrm{AB}$ oscillations; (ii) in order to observe the dephasing a finite bias on the detector is required but not sufficient; (iii) a complementary condition is that the imaginary part of the interaction selfenergy should be of the same order as the self-energy coming from the ring-dot coupling; (iv) if the above conditions are met the dephasing effect is more pronounced for weak ringdot couplings. The analysis we have performed shows that the visibility of the decoherence as the reduction of the AB oscillation does not depend on a single physical parameter whose magnitude should be carefully specified. The ratio of the imaginary parts of the two self-energies is a dimensionless quantity which can be adjusted by tuning the bias, the interaction strength and the ring-dot coupling. This is a useful freedom that one gains when considering two mutually coupled subsystems and justifies fully the setup proposed by Buks et al.

When the Keldysh formalism is employed to study transport through interacting systems having complex geometries a correction to the Landauer formula for the current does not allow a proper connection to the scattering theory. We have shown that this correction cannot be neglected since it exceeds the main contribution near the Fano dip. Moreover, as being entirely due to electron-electron interaction, it enhances the controlled dephasing. We hope our results will stimulate further theoretical and experimental work in this area.

\section{ACKNOWLEDGMENTS}

This research has been supported by the CEEX Grant No. 2976/2005. V.M. acknowledges the financial support from TUBITAK during the visit at Bilkent University. We thank A. Aldea for useful discussions. B. T. gratefully acknowledges support from TUBITAK and TUBA. 
${ }^{1}$ A. Yacoby, M. Heiblum, D. Mahalu, and H. Shtrikman, Phys. Rev. Lett. 74, 4047 (1995).

${ }^{2}$ R. Schuster, E. Bucks, M. Heiblum, D. Mahalu, V. Umanski, and H. Shtrikman, Nature (London) 385, 417 (1997).

${ }^{3}$ A. W. Holleitner, C. R. Decker, H. Qin, K. Eberl, and R. H. Blick, Phys. Rev. Lett. 87, 256802 (2001).

${ }^{4}$ K. Kobayashi, H. Aikawa, S. Katsumoto, and Y. Iye, Phys. Rev. Lett. 88, 256806 (2002); Phys. Rev. B 68, 235304 (2003).

${ }^{5}$ A. L. Yeyati and M. Büttiker, Phys. Rev. B 52, R14360 (1995).

${ }^{6}$ G. Hackenbroich and H. A. Weidenmüller, Phys. Rev. B 53, 16379 (1996).

${ }^{7}$ G. Hackenbroich, Phys. Rep. 343, 463 (2001).

${ }^{8}$ A. Aharony, O. Entin-Wohlman, and Y. Imry, Turk. J. Phys. 27, 299 (2003).

${ }^{9}$ O. Entin-Wohlman, A. Aharony, Y. Imry, and Y. Levinson, J. Low Temp. Phys. 126, 1251 (2002).

${ }^{10}$ W. Hofstetter, J. König, and H. Schoeller, Phys. Rev. Lett. 87, 156803 (2001).

${ }^{11}$ V. Moldoveanu, M. Țolea, A. Aldea, and B. Tanatar, Phys. Rev. B 71, 125338 (2005).

${ }^{12}$ V. Moldoveanu, M. Țolea, V. Gudmundsson, and A. Manolescu, Phys. Rev. B 72, 085338 (2005).

${ }^{13}$ J. Imry, Introduction to Mesoscopic Physics (Oxford University Press, Oxford, 1997).

${ }^{14}$ A. Stern, Y. Aharonov, and Y. Imry, Phys. Rev. A 41, 3436 (1990).

${ }^{15}$ S. A. Gurvitz, quant-ph/9607029 (unpublished), Phys. Rev. B 57, 6602 (1998).

${ }^{16}$ E. Buks, R. Schuster, M. Heiblum, D. Mahalu, and V. Umansky, Nature (London) 391, 871 (1998).

${ }^{17}$ D. Sprinzak, E. Buks, M. Heiblum, and H. Shtrikman, Phys. Rev. Lett. 84, 5820 (2000).

${ }^{18}$ I. L. Aleiner, N. S. Wingreen, and Y. Meir, Phys. Rev. Lett. 79,
3740 (1997).

${ }^{19}$ Y. Levinson, Europhys. Lett. 39, 299 (1997).

${ }^{20}$ R. P. Feynman and F. L. Vernon, Ann. Phys. (N.Y.) 24, 118 (1963).

${ }^{21}$ M. Büttiker and A. M. Martin, Phys. Rev. B 61, 2737 (2000).

${ }^{22}$ A. Silva and S. Levit, Phys. Rev. B 63, 201309(R) (2001).

${ }^{23}$ G. L. Khym, Y. Lee, and K. Kang, cond-mat/0601535 (unpublished).

${ }^{24}$ B. Szafran and F. M. Peeters, Phys. Rev. B 72, 165301 (2005).

${ }^{25}$ Z.-T. Jiang, Q.-f. Sun, X. C. Xie, and Y. Wang, Phys. Rev. Lett. 93, 076802 (2004).

${ }^{26}$ J. König and Y. Gefen, Phys. Rev. Lett. 86, 3855 (2001).

${ }^{27}$ H. Aikawa, K. Kobayashi, A. Sano, S. Katsumoto, and Y. Iye, Phys. Rev. Lett. 92, 176802 (2004).

${ }^{28}$ M. Avinun-Kalish, M. Heiblum, A. Silva, D. Mahalu, and V. Umansky, Phys. Rev. Lett. 92, 156801 (2004).

${ }^{29}$ A. Silva and S. Levit, Europhys. Lett. 62, 103 (2003).

${ }^{30}$ K. Kang, Phys. Rev. Lett. 95, 206808 (2005).

${ }^{31}$ M. Wagner, Phys. Rev. B 44, 6104 (1991).

${ }^{32}$ H. Haug and A.-P. Jauho, Quantum Kinetics in Transport and Optics of Semiconductors (Springer, Berlin, 1996).

${ }^{33}$ L. E. Henrickson, A. J. Glick, G. W. Bryant, and D. F. Barbe, Phys. Rev. B 50, 4482 (1994).

${ }^{34}$ D. C. Langreth, in Linear and Nonlinear Electron Transport in Solids, edited by J. T. Devreese and V. E. van Doren (Plenum Press, New York, 1976).

${ }^{35}$ A.-P. Jauho, N. S. Wingreen, and Y. Meir, Phys. Rev. B 50, 5528 (1994).

${ }^{36}$ Y. Meir and N. S. Wingreen, Phys. Rev. Lett. 68, 2512 (1992).

${ }^{37}$ J. M. Luttinger, Phys. Rev. 121, 942 (1961).

${ }^{38}$ A. Aldea, M.Țolea, and J. Zittartz, Physica E (Amsterdam) 28, 191 (2005). 